\title{
Experimental Identification of Kinematic Constraints
}

\author{
Pierre E. Dupont and Timothy M. Schulteis \\ pierre@bu.edu tschulte@bu.edu \\ Aerospace \& Mechanical Engineering \\ Boston University \\ Boston, MA 02215
}

\author{
Robert D. Howe \\ howe@deas . harvard .edu \\ Engineering \& Applied Sciences \\ Harvard University \\ Cambridge, MA 02138
}

\begin{abstract}
This paper proposes techniques for automatically identifying and modeling the kinematic constraints of manipulated objects. The existence of constraints is established by examination of the forces and torques normal and tangential to the object's motion. Constraints are modeled as joints with configuration and history dependent forces. To illustrate these concepts, several experimental examples are presented.
\end{abstract}

\section{Introduction}

The need to automatically determine the characteristics of manipulated objects arises in a number of applications. For example, in remediation of toxic waste dumps, quantitative measurements of the size and weight of the containers helps to infer their contents and to determine optimal handling strategies. Machine perception can also be used to develop models of objects for use in simulation, planning, and control. Such simulations have already been used to overcome stability problems related to teleoperation with significant time delays $[2,12]$. Similarly, the mechanical and visual aspects of virtual environment models can be automatically calibrated through manipulation of actual tools and objects which are appropriately instrumented.

In robotics, determination of object properties has focused on improving control performance. Parameter estimation techniques have been applied to the identification robot and payload lengths and inertias $[1,9,10]$, as well as to payload geometry and frictional characteristics [14]. Throughout this work, the kinematic constraints of the robot and payload are essentially specified a priori. This is because the kinematic constraints are used explicitly in the identification process. Constraints impose limits on the allowable configurations and velocities of the manipulated object, so when the constraints are known, reduced-order dynanic equations may be written. Parameter estimation is then framed as a search to determine the best parameter values that fit the dynamic equations with the given input-output data.

In an unstructured environment, constraints may not be known in advance. Their identification, however, is in many cases essential, because they reveal functional relationships among objects in the environment. For example, if the grasped object moves only in a circular trajectory and the dominant forces are in the radial direction, then the object is probably constrained to rotate about an axis, whose coordinates can be deduced from the observed motion. Similar inferences may be made about kinematic constraints from observing force-motion relationships for sliding or rolling objects. Identifying such constraints from manipulation data presents a new and difficult challenge that is different in character from the previous work on estimating the values of parameters such as lengths and inertias.

In this paper we explore the constraint identification process by examining and analyzing experimental data from two manipulation tasks. After reviewing background material on automatic parameter estimation and prior work on relevant areas, we describe a simple method for identifying kinematic constraints based on trajectory and force data. A method for modeling constraints is then proposed. These techniques are applied to a data from tasks where a two-fingered planar telemanipulator turns a crank and inserts a needle. We conclude with a discussion of extensions of the present techniques to more complex tasks.

\subsection{Background}

A system for automatic parameter identification would ideally have two types of irlput: some sort of high-level task description and the sensor data from execution of the task [14]. The output would be a quantitative description of the manipulated objects and their relationships to each other and the rest of the environment. The description that can be generated will obviously be limited to those properties that are exercised in execution of the task. For example, we cannot measure the friction coefficient of an object's surface if it never slides against another surface. Special exploratory motions might be required for full characterization. In any case, the intended use of the identified information will prescribe the information needed and usually the method to obtain it as well.

Given these inputs, the environment identification problem is composed of three main sub-problems: task decomposition, data segmentation, and parameter estimation 
[14]. The first step, task decomposition, involves describing the task and its constituent subtasks in terms of the mechanical phenomena which can be sensed during execution. The degree of detail which must be provided and the use of additional sensory information (such as vision) will depend on the application. In complex environments where many objects can interact in complicated ways, it may be impractical to enumerate all the possible interactions which may occur. In order to concentrate on the constraint identification problem, we assume in this paper that the task is simple and its decomposition into phases is given.

The next phase, data segmentation, is the reconstruction of the events or stages of a manipulation task based on streams of sensory data. This is important because the context or state of the system at any given time will dictate which parameters can be estimated at that time. For example, to find the coefficient of friction between the object and the manipulator, the estimator must determine when sliding is occurring. Segmentation of manipulation data has received considerable attention for skill transference from humans to autonomous robots $[8,13]$. In those studies, the goal was to find the commands which resulted in successful execution of each subtask, which may not be necessary for property estimation. Other work has been directed at determining the manipulation strategies of humans [11] or evaluating the performance of teleoperator systems $[4,5,6]$, where task decomposition can be used to relate performance to subtask attributes. For property estimation, segmentation may not require classification of every portion of the data stream.

Once the data is segmented in time, parameter estimation can proceed using sensory data within each of the segments, using techniques appropriate for that type of parameter. In the context of robotics, the identification of link lengths and inertial parameters has been thoroughly studied $[1,9]$. Geometric and frictional properties of manipulated objects have been estimated as well [3,14].

\section{Constraint Identification}

We are interested in identifying and modeling kinematic constraint forces. Ideally, kinematic constraints reduce the number of degrees of freedom of an object, as in rigid body contact. Figure 1 shows the trajectory of an object and the force vector on the object at one point along the path. If this object is undergoing pure constrained motion, then the component of the force normal to the direction of the instantaneous velocity $v, F_{n}$, is due to the constraint. $F_{t}$, the force in the direction of $v$, is due to other task-related interactions, such as frictional forces from sliding on the constraint surface. This suggests that a basic method of identifying constraint forces and separating other types of forces from them is decomposing the net force into components normal and parallel to the direction of motion.

In real tasks, non-constraint forces may of course act normal to the direction of motion. One simple example is gravity: if an object is carried horizontally, its weight

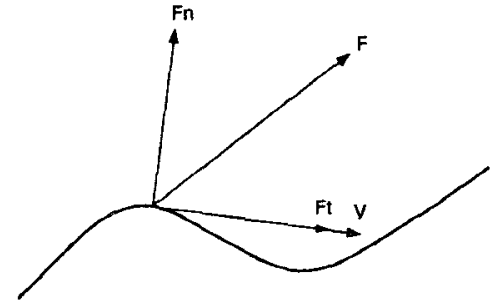

Figure 1: Vectors of force and velocity along trajectory of motion.

will appear in the vertical "constraint" direction normal to the motion. In a simple constraint identification system, disambiguating such non-constraint forces from actual constraints must rely on the task decomposition, which specifies the expected interactions. In more sophisticated systems, it will be possible to use contextual data from other parts of the task and other sources of information as well.

A number of other complicating factors arise in real tasks. In particular, the validity of the assumption of rigid constraints (i.e., no motion in the constrained direction) will depend on the compliance of both the manipulator and the object. This may require the consideration of the stiffness and damping at each interface. Furthermore, motion in the constrained as well as the unconstrained directions can be both configuration and history dependent. For example, under the Coulomb friction model, static friction may prevent an object from sliding until the friction force is exceeded. Before sliding, the object is completely constrained and no motion occurs, but once sliding begins a former constraint direction becomes a direction of motion. As a second example, consider the insertion of a needle through tissue and into a body cavity. Before contact with the tissue, motion is unconstrained. After contact, the insertion motion requires a force dependent on the insertion depth as tissues of different mechanical properties are traversed. When the body cavity is penetrated, the resistive force decreases abruptly. Finally, the force required to extract the needle may be configuration dependent as well.

The conclusion is that any general approach to modeling constraints must incorporate both configuration and history dependence for such properties as stiffness and damping. In addition, these properties may be nonlinear and only piecewise continuous.

\subsection{Approach}

Our investigation of constraint identification focuses on the experimental data presented below, where robot fingers grasp an object and execute a task. Our method for establishing the existence of kinematic constraints on the manipulated object requires the identification of the forces of constraint. In order to do so, the following assumptions are made:

- The forces and torques applied to the manipulated object by the fingers are known. 
- Knowledge of the finger trajectories (and the finger tip compliance) permits computation of the object trajectory.

- Task information specifies the major contributors to the net forces and torques applied to the manipulated object.

The approach to identifying constraint forces is as follows. By the first two assumptions, applied forces and torques are projected along the tangential and normal directions of motion obtained from velocity data. Based on the third assumption, dynamic equations are written in terms of the normal and tangential motion coordinates. Any unexplained forces or torques normal to the direction of motion are attributed to constraints. Occasionally, tasks involve the penetration of constraints, e.g., needle insertion. In these cases, the constraint is identified by unexplained forces or torques in the tangential direction. Data from repeated trials provide a means to refine force and displacement relationships and to determine if constraints are unilateral or bilateral. The examples in section 4 will clarify this procedure.

\section{Constraint Modeling}

Once the existence of a kinematic constraint has been established by detecting its forces and torques, it remains to be modeled. Modeling constraints and modeling mechanism motion have much in common. In fact, constraints can be expressed as joints with associated configurationdependent stiffness, damping, friction, etc. Recall from kinematics that multiple degree of freedom joints can be composed from joints which each possess a single degree of freedom. The three single degree of freedom joints are the revolute, prismatic and screw joints. Furthermore, screws with zero and infinite pitch (i.e., the ratio of translational to rotational motion) can be used to represent revolute and prismatic joints, respectively. This generality is exploited in screw theory in which the geometry of the screw is used as the basis for describing allowable instantaneous motion of a mechanism.

A screw is defined by its axis, a line in $\Re^{3}$, and by its pitch, which has units of length. In applying screw theory to kinematic chains composed of rigid links, the usual practice is to describe the instantaneous motion of a particular link as a linear combination of the configuration-dependent screws associated with the mechanism's joints. In this way, constraints do not explicitly appear in the set of screws or screw system describing the allowable motion.

Some readers may be familiar with wrenches which are coaxial force and moments acting along and about a screw axis. Wrenches are not used explicitly here since we choose to represent constraints as joints with configurationdependent forces. In particular, we model constraints as follows.

1. Constraints which are both rigid in comparison to the manipulator and configuration independent are mod-

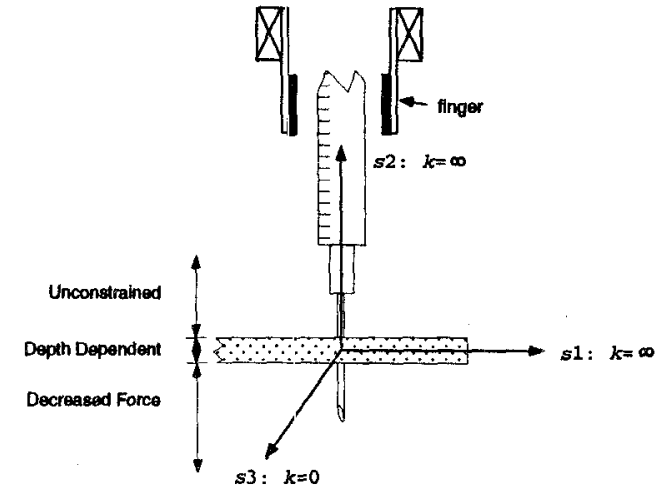

Figure 2: Screws describing constrained motion during needle insertion by a planar manipulator.

eled in the usual way by screws describing the allowable motion.

2. Constraints not meeting these conditions are modeled by screws in the constraint direction(s) which possess configuration-dependent properties, e.g., stiffness and damping.

Consider the insertion of a needle into tissue by a planar manipulator as shown in Figure 2. Experimental results for this example appear in the next section. This example is particularly interesting as the primary motion involves the penetration of a constraint surface. Only screws associated with the three planar degrees of freedom are shown since the constraints associated with out of plane motion are considered rigid.

Screws $s_{1}$ and $s_{2}$, in the horizontal and vertical directions, respectively, are of infinite pitch $(k=\infty)$ and represent allowable translations. Screw $s_{3}$ is of zero pitch $(k=0)$ and represents rotation in the plane. For any configuration of the needle in which contact is not made with the tissue, there are no constraint forces associated with the screws. As the needle penetrates the tissue, constraint forces and torque whaich are dependent on penetration depth, can be associated with $s_{1}, s_{2}$ and $s_{3}$.

\section{Experimental Examples}

We now apply these ideas to the data from two tasks. To avoid the necessity of programming the tasks for autonomous robotic execution, we used a planar, two-fingered teleoperated hand system controlled by a human operator [7]. This system trades a limitation on the number of joints for a clean and simple mechanical design. The system has high bandwidth and large dynamic range, which permits accurate control of contact forces and small motions. The system is designed to execute tasks that humans usually accomplish with a precision pinch grasp between the thumb and index finger (Fig. 3). The master and remote manipulators are kinematically identical, with two degrees of freedom in each finger, so finger tip position or force can be controlled within the vertical plane. The workspace is 

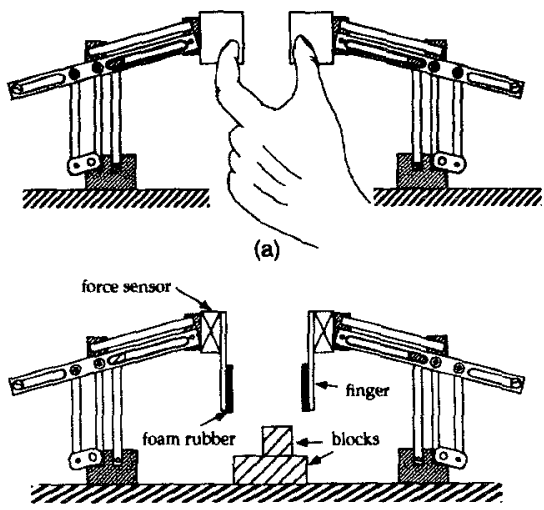

(b)

Figure 3: (a) Master manipulator with operator's hand. (b) Remote manipulator.

roughly circular and $75 \mathrm{~mm}$ in diameter. Parallelogram linkages maintain a constant vertical orientation of the fingertips. Flat, thin fingertips extending downward are mounted on the two remote manipulator fingers to facilitate manipulation of the objects used in the experiments (Fig. 3b). The manipulator fingertips were covered with a $2 \mathrm{~mm}$ layer of closed-cell foam rubber to increase compliance and friction. During each task, two-axis strain gauge force sensors measure contact forces on the remote manipulator finger tips, and joint sensors measured the finger tip positions and velocities.

\subsection{Constrained Versus Unconstrained Motion: Turning a Crank}

In the first example, the planar two-fingered hand is used to turn a crank and to move a block from one place on a table to another. The crank motion is constrained to lie on a circle (recorded trajectories are shown in Figure 4) while the free motion of the block is unconstrained. In both cases, examination of the data indicates that the motions were essentially quasi-static and inertial terms were negligible. Furthermore, the task decomposition (i.e., our a priori knowledge of the apparatus behavior) specifies that the component of the net force applied to the manipulated object which is normal to the direction of motion, $F_{n}$, will be dominated by gravity, $\left(F_{g}\right)_{n}$. Any remaining component of $F_{n}$ will be due to a constraint force, $F_{C}$.

$$
F_{n}=\left(F_{g}\right)_{n}+F_{C}
$$

The gravity forces of the crank and block were identified from the forces tangential to the motion. For example, from the task decomposition the force on the block tangential to the direction of motion, $F_{t}$, is expected to be of the form

$$
F_{t}=(m g)_{t}+\mu F_{n}+c v
$$

where $(m g)_{t}$ is the component of gravity tangential to the motion, $\mu$ is a coefficient of friction, $c$ is a viscous coefficient and $v$ is the object velocity. In Figure $5, F_{t}$ for the crank is plotted along with its least squares approximation.

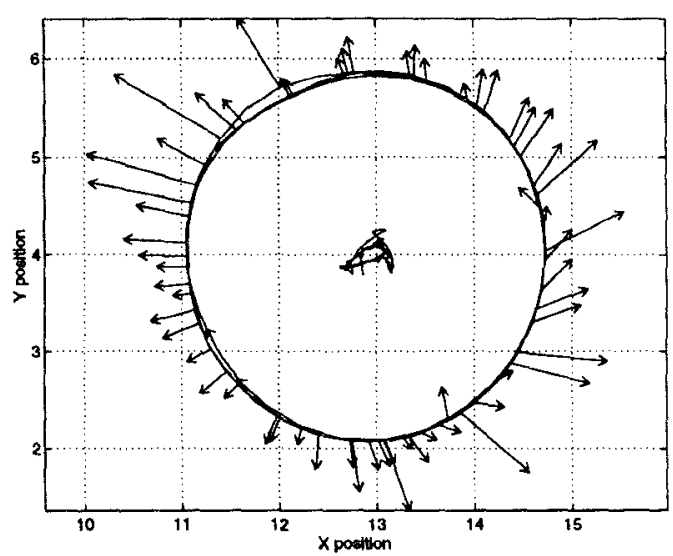

Figure 4: Position of crank handle, vectors of applied force, and computed center of rotation. The vectors show direction and magnitude of force applied by the robot hand.

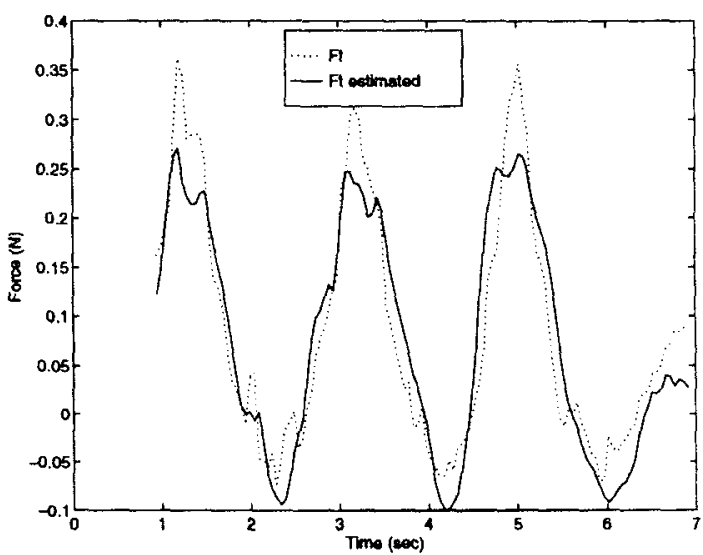

Figure 5: Tangential force, $F_{t}$, for the crank and the least squares approximation given by $m g=0.16 \mathrm{~N}, \mu=0.053$ and $c=0.010$

To test for the presence of constraints, $F_{n}-\left(F_{g}\right)_{n}$ is plotted for the crank and block motions in Figure 6. As expected, the constraint force on the block is negligible during its free motion while a significant positive constraint force can be seen throughout for the crank. The sign of the constraint force indicates that the operator was pulling radially outward on the crank while turning it. While the crank represents a bilateral radial constraint, the motion of the operator was insufficient to fully capture this fact. This example shows how exploratory motions or repeated trials by different operators may be necessary to fully identify a constraint.

The crank handle and bearings are much stiffer than the manipulator finger pads. In addition, the constraint is active for all configurations of the crank. Thus, we employ rule (1) from section 3 and use a single screw of zero pitch to describe the constraint. The location of the screw axis in the plane of motion is equivalent to the center of rotation. The center of rotation is computed by intersecting normals to the instantaneous direction of motion for 12 consecutive 


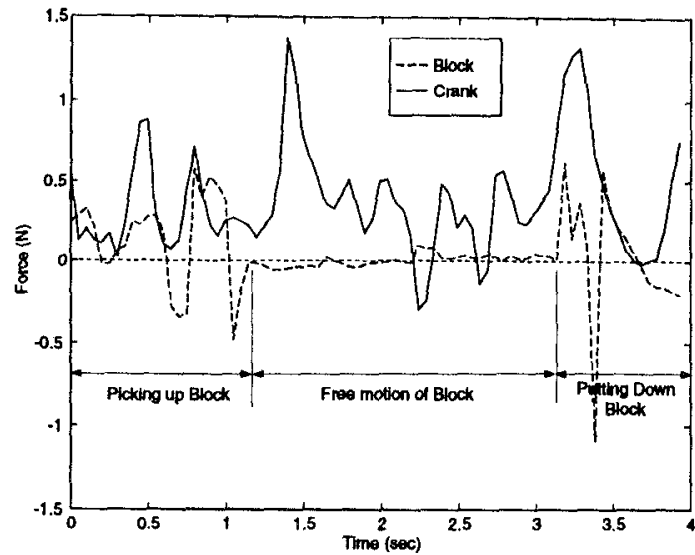

Figure 6: Constraint forces, $F_{C}$, for the block and crank.

data points and then finding their mean position. Figure 4 depicts the position of the crank handle computed as the average displacement of the fingers as well as the center of rotation for three rotations of the crank and the force vectors applied by the fingers. Drift in the location of the screw axis is largely due to slippage between the fingers and the crank handle. Finger stiffness is relatively unimportant here, as it only accounts for displacements of about 0.11 $\mathrm{cm}$. Note that if the fingers were grasping the coupler link of a four-bar mechanism, the location in the plane of the screw axis would be configuration dependent. Also note that the applied force when moving upward (right half of circle) has a distinct tangential component, but when moving downward the applied force has a very small tangential component. This is due to the fact that gravity and friction forces are additive while moving up but almost negate each other while going down.

\subsection{Configuration Dependent Constraints: Needle Puncture}

In this example, a hypodermic needle was inserted through the bottom of an inverted paper cup as shown in Figure 2 using the manipulator of Figure 3. Since the constraints are both configuration and history dependent, they are modeled using rule 2 of section 3 . In particular, we focus on the constraint force in the direction of needle insertion which is associated with screw $s_{2}$. The screw axis itself can be located using position and velocity data from the fingers.

As in the previous example, the insertion motion was quasi-static. Since the constraint force lies in the direction of motion, the tangential force equation becomes

$$
F_{t}=\left(F_{g}\right)_{t}+F_{C}
$$

the force $F_{C}$ is plotted along with tangential displacement in Figure 7. The subtasks of free motion, needle insertion, penetration and extraction can be clearly seen in the data.

The history dependence of the constraint force arises from two factors. First, the force depends on the maximum

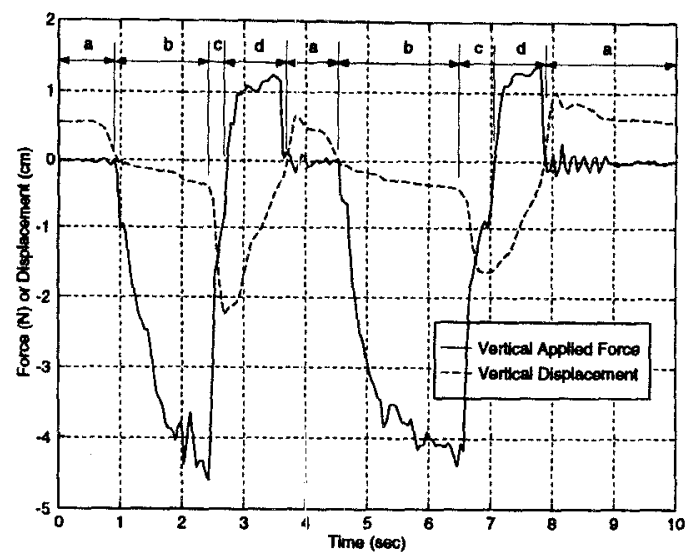

Figure 7: Constraint force and displacement in insertion direction. $a=$ free motion, $b=$ insertion, $c=$ penetration, $d=$ extraction.

prior insertion depth. This can be seen by the fact that the extraction force is much smaller than the insertion force. Secondly, the cup bottom acts as an elastic membrane in series with the elastic fingerpads. During extraction, the constraint force depends on the displacement of both from their relaxed positions. A model of constraint stiffness can be obtained by dividing the constraint force by the position at each point in time. The history dependent stiffness as a function of time is plotted in Figure 8 .

Repeated trials with direction reversals at various depths would allow for the identification of a more detailed model.

\section{Conclusion}

Kinematic constraints impose limits on the allowable configurations and velocities of an object. Traditionally, constraints are used to relate the motions of rigid bodies, but they are also useful because they provide information about the functional relationships among objects in unstructured environments. In this paper we described simple methods for identifying and modeling unknown, compliant kinematic constraints on a manipulated object. Constraints are identified, in general, by examining the forces and torques directed normal to motion. We propose modeling constraints as joints with configuration-dependent properties. The experimental results presented here successfully demonstrate these concepts for two simple planar systems, including objects that changed mechanical configuration (i.e. puncture) during task execution.

This paper has only begun to examine the many issues that must be addressed for a complete automatic constraint identification system. One essential question is the degree of automation that can be achieved. This will be strongly dependent on the task decomposition, and among the key questions is how much detail must be specified by the human programmer, and what representation will be most appropriate. 

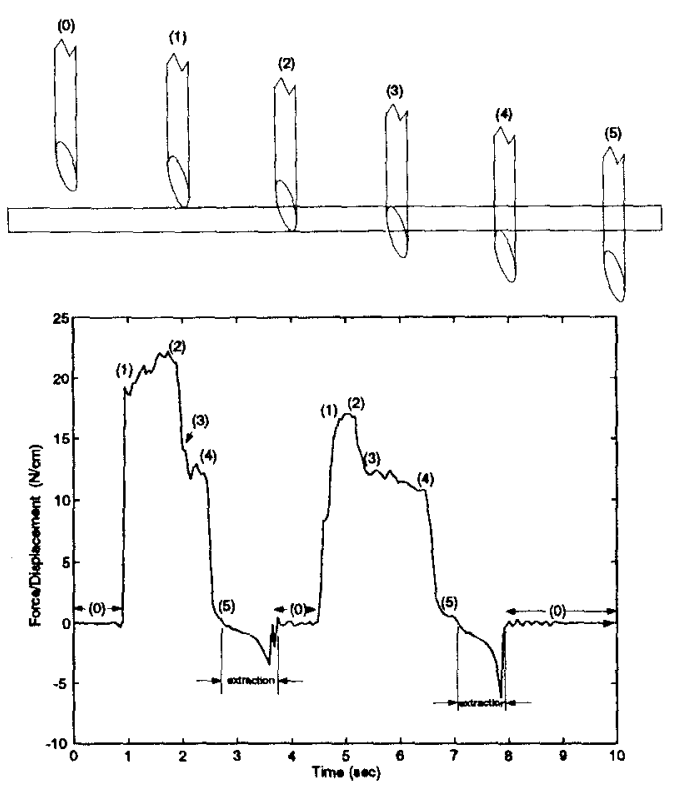

Figure 8: Model of history dependent stiffness. The position of the needle tip corresponding to each number on the plot shows configuration information at key transition points in the insertion process.

For modeling more complex systems, particularly mechanisms, we are investigating the connection between the constraint identification problem and kinematic synthesis algorithms. In both cases the motion-force relationship is specified and the kinematic structure is to be found. This analogy may permit immediate application of the extensive techniques developed in previous kinematics research.

\section{Acknowledgments}

The authors would like to thank Paul Millman for helpful discussions and assistance with the experiments reported here. This work was partially supported by the Office of Naval Research under Grant No. N00014-92-J1877.

The views expressed in this article are those of the authors and do not reflect the official policy or position of the United States Air Force, Department of Defense, or the US Government. Approved for public release; distribution unlimited.

\section{References}

1. An, C., Atkeson, C., and Hollerbach, J., "Estimation of Inertial Parameters of Rigid Body Links of Manipulators." Proc. 24th Conference on Decision and Control, Ft. Lauderdale, FL, December 1985, pp. 990995.

2. Bejczy, A. K., Kim, W. S., and Venema, S. C., 1990, "The Phantom Robot: Predictive Displays for Teleoperation with Time Delay," Proceedings of the 1990
IEEE International Conference on Robotics and Automation, Vol. 1, May, pp. 546-551.

3. Fyler, D., 1981, "Computer Graphic Representation of Remote Environments Using Position Tactile Sensors," SM thesis, MIT, August.

4. Hannaford, B., and Lee, P., 1991, "Hidden Markov Modal Analysis of Force/Torque Information in Telemanipulation," International Journal of Robotics Research, Vol. 10, No. 5, October, pp. 528-539.

5. Hannaford, B., Wood, L., Guggisberg, B., McAffee, D., and Zak, H., 1989, "Performance Evaluation of a Six-Axis Generalized Force-Reflecting Teleoperator," JPL Publication 89-18, Pasadena, CA.

6. Hannaford, B., Wood, L., McAffee, D. A., and Zak, H., 1991, "Performance Evaluation of a Six-Axis Generalized Force-Reflecting Teleoperator," IEEE Transactions on Systems, Man, and Cybernetics, Vol. 21, No. 3, May/June, pp. 620-633.

7. Howe, R. D., 1992, "A Force-Reflecting Teleoperated Hand System for the Study of Tactile Sensing in Precision Manipulation," Proceedings of the 1992 IEEE International Conference on Robotics and Automation, Nice, France, Vol. 2., May, pp. 1321-1326.

8. Kang, S. B., and Ikeuchi, K., 1993, "Toward Automatic Robot Instruction from Perception - Recognizing a Grasp From Observation," IEEE Transactions on Robotics and Automation, Vol. 9, No. 4, pp. 432443.

9. Khosla, P., and Kanade, T., 1985, "Parameter Identification of Robot Dynamics." Proceedings of the 24th Conference on Decision and Control, Ft. Lauderdale, FL, December, pp. 1754-1760.

10. Lin, S. T., and Yae, K. H., 1992, "Identification of Unknown Payload and Environmental Parameters for Robot Compliant Motion" Proceedings of the 1992 American Control Conference, Chicago, Vol. 4, June, pp. 2952-2956.

11. McCarragher, B. J., 1994, "Force Sensing from Human Demonstration Using a Hybrid Dynamical Model and Qualitative Reasoning," Proceedings of the 1994 IEEE Conference on Robotics and Automation, Vol. 1, May, pp. 557-563.

12. Noyes, M. V. and Sheridan, T. B. 1984. "A Novel Predictor for Telemanipulation through a Time Delay," Proceedings of the Annual Conference on Manual Control, Moffett Field, CA, NASA Ames Research Center.

13. Pook, P. K., and Ballard, D. H., 1993, "Recognizing Teleoperated Manipulations," Proceedings of the IEEE International Conference on Robotics and Automation, Vol. 2, May, pp. 578-585.

14. Schulteis, T. M., Dupont, P. E., Millman P. A., and Howe, R. D., 1996, "Automatic Identification of Remote Environments" Proceedings of the ASME Dynamic Systems and Control Division, Atlanta, DSCVol. 58, November, pp. 451-458. 